\title{
Improved Theory of the Muonium Hyperfine Structure
}

\author{
T. Kinoshita and M. Nio \\ Newman Laboratory of Nuclear Studies, Cornell University, Ithaca, NY 14853
}

\begin{abstract}
Terms contributing to the hyperfine structure of the muonium ground state at the level of few tenths of $\mathrm{kHz}$ have been evaluated. The $\alpha^{2}(Z \alpha)$ radiative correction has been calculated numerically to the precision of $0.02 \mathrm{kHz}$. Leading $\ln (Z \alpha)$ terms of order $\alpha^{4-n}(Z \alpha)^{n}, n=1,2,3$, and some relativistic corrections have been evaluated analytically. The theoretical uncertainty is now reduced to $0.17 \mathrm{kHz}$. At present, however, it is not possible to test QED to this precision because of the $1.34 \mathrm{kHz}$ uncertainty due to the muon mass. PACS numbers: 36.10.Dr, 12.20.Ds, 31.30.Jv, 06.20.Jr
\end{abstract}

Typeset using REVTEX 
The hyperfine splitting of the muonium ground state is one of very precisely measured quantities [1]:

$$
\Delta \nu(\exp )=4463302.88(16) \mathrm{kHz} \quad(0.036 \mathrm{ppm}) .
$$

Currently new experiment is in progress to improve the measurement of $\Delta \nu(\exp )$ and muon mass by a factor of five or more [2]. This is very important for testing the validity of quantum electrodynamics (QED) since $\Delta \nu$ can be calculated very precisely in QED, being relatively free from the effect of hadronic interaction. The precision of such a test is limited at present by the uncertainty in theoretical calculation, which may exceed $1 \mathrm{kHz}$. This paper reports our result in which we have reduced this uncertainty by nearly an order of magnitude.

As is well known, the bulk of the hyperfine splitting is given by the Fermi formula

$$
E_{F}=\frac{16}{3}(Z \alpha)^{2} c R_{\infty} \frac{m_{e}}{m_{\mu}}\left[1+\frac{m_{e}}{m_{\mu}}\right]^{-3}
$$

where $Z$ is the charge of the muon in units of the electron charge, $R_{\infty}$ is the Rydberg constant for infinite nuclear mass, and $m_{e}$ and $m_{\mu}$ are the electron and muon masses, respectively. Of course $Z=1$ for the muon, but it is kept in the formula in order to distinguish the contribution of binding effect $(\mathrm{Z} \alpha)$ from that of radiative correction $(\alpha)$.

Many correction terms of both $\alpha$ and Z $\alpha$ type have been calculated over 40 years. It is customary to classify them into three types: radiative non-recoil correction, pure recoil correction, and radiative-recoil correction. In addition there is a small weak interaction contribution. Thus one may write

$$
\Delta \nu(\text { theory })=\Delta \nu(\operatorname{rad})+\Delta \nu(\text { recoil })+\Delta \nu(\operatorname{rad}-\text { recoil })+\Delta \nu(\text { weak })
$$

Conventionally, the effect of hadronic vacuum polarization is included in $\Delta \nu$ (rad-recoil).

Purely radiative terms of orders $\alpha(Z \alpha)$ and $\alpha(Z \alpha)^{2}$ have been known for some time [3]:

$$
\begin{aligned}
\Delta \nu(\mathrm{rad})= & \left(1+a_{\mu}\right)\left(1+\frac{3}{2}(Z \alpha)^{2}+a_{e}+\alpha(Z \alpha)\left(\ln 2-\frac{5}{2}\right)\right. \\
& -\frac{8 \alpha(Z \alpha)^{2}}{3 \pi} \ln (Z \alpha)\left[\ln (Z \alpha)-\ln 4+\frac{281}{480}\right] \\
& \left.+\frac{\alpha(Z \alpha)^{2}}{\pi}(15.38 \pm 0.29)\right) E_{F} .
\end{aligned}
$$


Here $a_{e}$ and $a_{\mu}$ are the anomalous magnetic moments of the electron and muon, respectively. The appearance of the factor $\left(1+a_{\mu}\right)$ in (4) is in accord with our definition of $E_{F}$ in (2). The known recoil corrections add up to [3]

$$
\begin{aligned}
\Delta \nu(\text { recoil }) & =\left(-\frac{3 Z \alpha}{\pi} \frac{m_{e} m_{\mu}}{m_{\mu}^{2}-m_{e}^{2}} \ln \frac{m_{\mu}}{m_{e}}\right. \\
& \left.+\frac{\gamma^{2}}{m_{e} m_{\mu}}\left[2 \ln \frac{m_{r}}{2 \gamma}-6 \ln 2+\frac{65}{18}\right]\right) E_{F},
\end{aligned}
$$

where $\gamma \equiv Z \alpha m_{r}, m_{r}=m_{e} m_{\mu} /\left(m_{e}+m_{\mu}\right)$. The radiative-recoil contributions, which arise from both lepton lines and vacuum polarizations, are given by

$$
\begin{aligned}
\Delta \nu(\text { rad-recoil }) & =\frac{\alpha(Z \alpha)}{\pi^{2}} \frac{m_{e}}{m_{\mu}}\left(-2 \ln ^{2} \frac{m_{\mu}}{m_{e}}+\frac{13}{12} \ln \frac{m_{\mu}}{m_{e}}\right. \\
& +\frac{21}{2} \zeta(3)+\zeta(2)+\frac{35}{9}+(2.15 \pm 0.14) \\
& \left.+\frac{\alpha}{\pi}\left[-\frac{4}{3} \ln ^{3} \frac{m_{\mu}}{m_{e}}+\frac{4}{3} \ln ^{2} \frac{m_{\mu}}{m_{e}}+\mathcal{O}\left(\ln \frac{m_{\mu}}{m_{e}}\right)\right]\right) E_{F},
\end{aligned}
$$

The $\alpha(Z \alpha)$ term is known exactly [3, [4] except for the hadronic vacuum polarization contribution (the $(2.15 \pm 0.14)$ term) [5]. The $\ln ^{3}$ and $\ln ^{2}$ parts of the $\alpha^{2}(Z \alpha)$ term were evaluated by Eides et al. [6]. Finally there is a small contribution due to the $Z^{0}$ exchange. Our re-evaluation of the standard-model estimate [7] gives

$$
\Delta \nu(\text { weak }) \simeq 0.065 \mathrm{kHz} .
$$

As is clear from these results one must know the $\alpha^{2}(Z \alpha)$ radiative correction in order to improve the theoretical prediction further. Fig. 1 shows typical diagrams contributing to this order. Recently, terms represented by the diagrams (a) - (e) of Fig. 1 have been evaluated by Eides et al. [8]. Their results are as follows:

$$
\begin{aligned}
\Delta \nu(\text { Fig.1(a) }) & =\frac{36}{35} \frac{\alpha^{2}(Z \alpha)}{\pi} E_{F} \\
& =0.567 \mathrm{kHz}, \\
\Delta \nu(\text { Fig.1(b) }) & =\left(\frac{224}{15} \ln 2-\frac{38}{15} \pi-\frac{118}{225}\right) \frac{\alpha^{2}(Z \alpha)}{\pi} E_{F} \\
& =1.030 \mathrm{kHz},
\end{aligned}
$$




$$
\begin{aligned}
& \Delta \nu(\text { Fig. } 1(\mathrm{c}))=\left(-\frac{4}{3} z^{2}-\frac{20 \sqrt{5}}{9} z-\frac{64}{45} \ln 2+\frac{\pi^{2}}{9}+\frac{1043}{675}+\frac{3}{8}\right) \frac{\alpha^{2}(Z \alpha)}{\pi} E_{F} \\
& =-0.369 \mathrm{kHz} \text {, } \\
& \Delta \nu(\text { Fig. } 1(\mathrm{~d}))=-0.310742 \cdots \frac{\alpha^{2}(Z \alpha)}{\pi} E_{F} \\
& =-0.171 \mathrm{kHz} \text {, }
\end{aligned}
$$

where $z=\ln ((1+\sqrt{5}) / 2)$. The results $(8),(9)$ and $(\sqrt{10})$ are analytic, while (11) was evaluated numerically after reducing the integral to one dimension. We confirmed these results by an independent numerical calculation. However, our purely numerical evaluation of Fig. 1(e):

$$
\begin{aligned}
\Delta \nu(\text { Fig.1(e) }) & =-0.47248(9) \frac{\alpha^{2}(Z \alpha)}{\pi} E_{F} \\
& =-0.261 \mathrm{kHz}
\end{aligned}
$$

disagrees with the semi-analytic result of Ref. [9]. Recently Eides [10 found an error in the Table after Eq. (23) of Ref. 9]. Their corrected value is in good agreement with (12).

Fig. 2 shows the complete set of Feynman diagrams of type (f) of Fig. 1, which has not yet been evaluated. The primary purpose of this paper is to report a preliminary result of our calculation for all diagrams of Fig. 2 carried out in the Feynman gauge:

$$
\begin{aligned}
\Delta \nu(\text { Fig.1(f) }) & =(-0.63 \pm 0.04) \frac{\alpha^{2}(Z \alpha)}{\pi} E_{F} \\
& =-0.347(0.022) \mathrm{kHz},
\end{aligned}
$$

where the error is mainly due to the uncertainty in extrapolating the integral to zero infrared cutoff. Details of calculation will be reported elsewhere. The complete $\alpha^{2}(Z \alpha)$ correction is the sum of (8) - (11), (12), and (13):

$$
\Delta \nu(\text { Fig. } 1)=0.449(0.022) \mathrm{kHz}
$$

Recently we have received two preprints from Eides and his collaborators [11], which report the result of their calculation, carried out in the Fried-Yennie gauge, for part of the diagrams of Fig. 2. We were able to compare our result for the sum of diagrams H17, H18 
and H19 with theirs since this sum is invariant under the covariant gauge transformation. They are in perfect agreement although individually they have quite different values due to different gauges. To compare other diagrams, we have to wait for completion of their calculation.

The remaining theoretical uncertainty in $\Delta \nu$ comes mainly from terms of orders $\alpha^{4}$ and $\alpha^{3}\left(m_{e} / m_{\mu}\right)$. Although these diagrams are of higher order than (14), they may have numerically comparable magnitudes due to the appearance of $\ln (Z \alpha)$ and/or $\ln \left(m_{\mu} / m_{e}\right)$ factors. Some of these contributions are known: [12]

$$
\begin{aligned}
\delta \Delta \nu= & -\frac{8}{3}\left(\frac{\alpha}{2 \pi}-2 \frac{m_{e}}{m_{\mu}}+\frac{Z}{4} \frac{m_{e}}{m_{\mu}}\right) \frac{\alpha}{\pi}(Z \alpha)^{2} \ln ^{2}(Z \alpha) E_{F} \\
& +\frac{17}{8}(Z \alpha)^{4} E_{F} \\
= & 0.287 \mathrm{kHz}
\end{aligned}
$$

where the first three terms come from the magnetic form factor correction to the $\delta$-function potential $V_{F}$ whose expectation value is $E_{F}$, the reduced mass correction to the $\ln ^{2}(Z \alpha)$ terms of (4), and the ln $k$ part of the Salpeter term of the Lamb shift [13, respectively. The last term is a higher order Breit correction.

Here we report additional terms evaluated in the NRQED perturbation theory [14:

$$
\begin{aligned}
& \Delta \nu(\alpha)=<V_{50} G V_{F}>+\cdots, \\
& \Delta \nu(\beta)=<V_{2-\text { loop }} G V_{F}>+\cdots, \\
& \Delta \nu(\gamma)=<V_{S} G V_{F}>+\cdots, \\
& \Delta \nu(\delta)=<V_{\mathrm{hfs}} G(K \text { or } D)>+\cdots, \\
& \Delta \nu(\epsilon)=a_{e}<V_{40} G V_{F}>+\cdots,
\end{aligned}
$$

where $\langle\cdots\rangle$ means the difference of the triplet and singlet expectation values with respect to the non-relativistic wave function of the muonium ground state. $G$ is the Green's function of the non-relativistic electron in the muon Coulomb potential, and $D$ and $K$ are the Darwin and $k^{4}$-kinetic energy term. $V_{50}$ and $V_{2-\text { loop }}$ are $\delta$-function potentials whose expectation 
values are the $A_{50}$ term of the Lamb-shift energy with one virtual photon spanning over any number of Coulomb photons [15] and the term arising from two spanning photons as well as the vacuum-polarization effect [16], respectively. $V_{S}$ is the $\delta$-function potential corresponding to the part of the Salpeter term not included in (15). $V_{\mathrm{hfs}}$ is the effective hyperfine interaction potential corresponding to the $\ln \left(m_{\mu} / m_{e}\right)$ term of (5)). The last term of (16) comes from the $A_{40}$ part of the Lamb shift [15] and the electron anomaly correction to $V_{F}$.

All these contributions have been evaluated analytically. Their numerical values are

$$
\begin{gathered}
\Delta \nu(\alpha)=-0.381 \mathrm{kHz}, \\
\Delta \nu(\beta)=-0.007 \mathrm{kHz}, \\
\Delta \nu(\gamma)=-0.189 \mathrm{kHz}, \\
\Delta \nu(\delta)=-0.210 \mathrm{kHz}, \\
\Delta \nu(\epsilon)=0.004 \mathrm{kHz} .
\end{gathered}
$$

The term $\Delta \nu(\alpha)$ is of order $\alpha(Z \alpha)^{3} \ln (Z \alpha)$ and was obtained by Lepage [17]. The term $\Delta \nu(\beta)$ is of order $\alpha^{2}(Z \alpha)^{2} \ln (Z \alpha)$. Unfortunately, this evaluation is incomplete since $V_{2-\text { loop }}$ is not yet fully known. However, the contribution of remaining terms will not be much larger than the above result. The term $\Delta \nu(\gamma)$ is of order $\left(m_{e} / m_{\mu}\right)(Z \alpha)^{3} \ln (Z \alpha) . \Delta \nu(\delta)$ and $\Delta \nu(\epsilon)$ are proportional to $(Z \alpha)^{3} \ln (Z \alpha)\left(m_{e} / m_{\mu}\right) \ln \left(m_{\mu} / m_{e}\right)$ and $\alpha^{2}(Z \alpha)^{2} \ln (Z \alpha)$, respectively.

The terms (15) and (17) add up to $-0.496 \mathrm{kHz}$. The uncertainty due to uncalculated terms will be about $0.05 \mathrm{kHz}$. Including these estimates and using the value of $\alpha, R_{\infty}$ and $m_{\mu} / m_{e}$ from Refs. [18], [19] and [1]:

$$
\begin{aligned}
\alpha^{-1} & =137.0359979(32) \quad(0.024 \mathrm{ppm}) \\
R_{\infty} & =10973731.56830(31) \mathrm{m}^{-1} \\
\frac{m_{\mu}}{m_{e}} & =206.768259(62)
\end{aligned}
$$

we find 


$$
\Delta \nu \text { (theory) }=4463302.63(1.34)(0.21)(0.17) \mathrm{kHz},
$$

where the first and second errors reflect the uncertainties in the measurements of $m_{\mu}$ and $\alpha^{-1}$ listed in (18). The third error is purely theoretical and dominated by the uncertainty in the last $\alpha(Z \alpha)^{2}$ term of (耳). Further reduction of this error is very important and will be attempted shortly. The agreement between $\Delta \nu$ (theory) and $\Delta \nu(\exp )$ is excellent, the difference being

$$
-0.26(0.16)(1.34)(0.21)(0.17) \mathrm{kHz},
$$

where the first error is from the experiment (四) and the rest are carried over from (19).

The result (19) is obtained using the value of $\alpha$ from (18) which is determined from the quantum Hall effect. Actually, a more accurate value of $\alpha$ is known from the theory and measurement of the electron anomalous magnetic moment, which is 20]

$$
\alpha^{-1}=137.03599222(94) \text {. }
$$

If one uses this instead of (18), one finds

$$
\Delta \nu(\text { theory })^{\prime}=4463303.00(1.34)(0.06)(0.17) \mathrm{kHz} .
$$

Alternately, if one assumes that the uncertainty due to $\alpha$ is $0.06 \mathrm{kHz}$ and that QED is correct to $0.17 \mathrm{kHz}$, one can determine the muon mass from (11) and (22). This leads to

$$
\frac{m_{\mu}}{m_{e}}=206.768275(11)
$$

which is 5.6 times more accurate than the value quoted in (18). This precision is close to that expected from the new direct measurement of $m_{\mu}$ [2]. With the new measurement of muonium hyperfine structure and further improvement of theory, it will be possible to replace (23) by an even better one. Comparison of this result with the directly measured $m_{\mu}$ may be regarded as an alternative way to test the validity of QED. 


\section{ACKNOWLEDGMENTS}

We thank G. P. Lepage, P. Labelle and late D. R. Yennie for useful discussions. Thanks are due to M. I. Eides for communicating their preliminary result and pointing out some misprints and oversights in our paper. We thank the hospitality of the National Laboratory for High Energy Physics (KEK), Japan, where T. K. was visiting on sabbatical leave from Cornell University and M. N. was a short term visitor. This research is supported in part by the U. S. National Science Foundation. Part of numerical work was conducted at the Cornell National Supercomputing Facility, which receives major funding from the US National Science Foundation and the IBM Corporation, with additional support from New York State and members of the Corporate Research Institute. 


\section{REFERENCES}

[1] F. G. Mariam et al., Phys. Rev. Lett. 49, 993 (1982); E. Klempt et al., Phys. Rev. D25, 652 (1982).

[2] V. W. Hughes and G. zu Putlitz, Comm. Nucl. Part. Phys. 12, 259 (1984).

[3] J. R. Sapirstein and D. R. Yennie, in Quantum Electrodynamics, ed. by T. Kinoshita (World Scientific, Singapore,1990), pp. 560 - 672.

[4] M. I. Eides, S. G. Karshenboim, and V. A. Shelyuto, Ann. Phys. 205, 231 (1991); 205, 291 (1991); M. I. Eides, S. G. Karshenboim, and V. A. Shelyuto, Phys. Lett. 202B, 572 (1988).

[5] A. Karimkhodzhaev and R. N. Faustov, Yad. Fiz. 53, 1012 (1991) [Eng. transl.: Sov. J. Nucl. Phys. 53, 626 (1991)].

[6] M. I. Eides, S. G. Karshenboim, and V. A. Shelyuto, Phys. Lett. B216, 405 (1989); M. I. Eides and V. A. Shelyuto, Phys. Lett. B146, 241 (1984).

[7] M. A. B. Bég and G. Feinberg, Phys. Rev. Lett. 33, 606 (1974); G. T. Bodwin and D. R. Yennie, Phys. Rep. 43C, 267 (1978).

[8] M. I. Eides, S. G. Karshenboim, and V. A. Shelyuto, Phys. Lett. B229, 285 (1989); B249, 519 (1990).

[9] M. I. Eides, S. G. Karshenboim, and V. A. Shelyuto, Yad. Fiz. 55, 466 (1992) [Eng. transl.: Sov. J. Nucl. Phys. 55, 257 (1992)]; Phys. Lett. B268, 433 (1991); Phys. Lett. B316, 631(E) (1993).

[10] M. I. Eides, S. G. Karshenboim, and V. A. Shelyuto, Phys. Lett. B319, 545(E) (1993).

[11] M. I. Eides, S. G. Karshenboim, and V. A. Shelyuto, Phys. Lett. B312, 358 (1993) and Penn State U. preprint PSU/TH/129, 1993; M. I. Eides, S. G. Karshenboim, and V. A. Shelyuto, Petersburg Nuclear Physics Institute preprint PNPI-1942, 1993. 
[12] S. G. Karshenboim, Zh. Eksp.Teor. Fiz. 103, 1105 (1993) [Eng. transl.: JETP 76, 541 (1993)].

[13] E. E. Salpeter, Phys. Rev. 87, 328 (1952).

[14] W. E. Caswell and G. P. Lepage, Phys. Lett. B167, 437 (1986).

[15] See Ref. [3], p.569.

[16] See Ref. [3], p.572.

[17] Private communication from G. P. Lepage.

[18] M. E. Cage et al., IEEE Trans. Instrum. Meas., IM-38, 284 (1989).

[19] F. Nez et al., Phys. Rev. Lett. 69, 2326 (1992); M. Weitz et al., Phys. Rev. Lett. 72, 328 (1994).

[20] T. Kinoshita and D. R. Yennie, in Quantum Electrodynamics, ed. by T. Kinoshita (World Scientific, Singapore,1990), pp. 1 - 14. 


\section{FIGURES}

FIG. 1. Representative diagrams contributing to the $\alpha^{2}(Z \alpha)$ radiative corrections to the muonium hyperfine structure in which two virtual photons are exchanged between $e^{-}$and $\mu^{+}$. The muon is represented by $\times$.

FIG. 2. Two-photon exchange diagrams with fourth-order radiative corrections on the electron

line. Diagrams which are related to these diagrams by time reversal are not shown explicitly. The muon is represented by $\times$. 
This figure "fig1-1.png" is available in "png" format from: http://arxiv.org/ps/hep-ph/9402260v2 
This figure "fig1-2.png" is available in "png" format from: http://arxiv.org/ps/hep-ph/9402260v2 